\title{
Cost Effectiveness of Guanfacine Extended Release as an Adjunctive Therapy to a Stimulant Compared with Stimulant Monotherapy for the Treatment of Attention-Deficit Hyperactivity Disorder in Children and Adolescents
}

Vanja Sikirica, ${ }^{1}$ M. Haim Erder, ${ }^{1}$ Jipan Xie, ${ }^{2}$ Dendy Macaulay, ${ }^{2}$ Melissa Diener, ${ }^{2}$ Paul Hodgkins ${ }^{1}$ and Eric Q. Wu ${ }^{3}$

1 Shire Development LLC., Wayne, PA, USA

2 Analysis Group, Inc., New York, NY, USA

3 Analysis Group, Inc., Boston, MA, USA

Background: Attention-deficit hyperactivity disorder (ADHD) is a common psychiatric disorder in childhood, affecting 3-7\% of school-age children in the US and imposing substantial economic burden. Stimulants are considered first-line pharmacological treatment and are the most prescribed treatment for ADHD. However, approximately $30 \%$ of children with ADHD do not have an optimal response to a single stimulant and may require adjunctive therapy.

Objective: Our objective was to conduct a cost-effectiveness analysis (CEA) of adding a non-stimulant, guanfacine extended release (GXR), to stimulants versus maintaining existing stimulant monotherapy in the treatment of ADHD in children and adolescents with suboptimal response to stimulant monotherapy.

Methods: A 1-year Markov model was developed to estimate costs and effectiveness from a US third-party payer perspective. Effectiveness was measured by the QALY. The model assumed that patients transitioned among four health states (normal, mild, moderate and severe), defined by the Clinical Global Impression-Severity (CGI-S) scale. Transition probabilities were estimated in an ordered logit model using patient-level data from a multicentre, 9-week, double-blind, placebo-controlled, dose-optimization study, where subjects $(n=461)$ continued their stable morning stimulant and were randomized to GXR administered in the morning, GXR administered in the evening, or placebo. The model assumed that patients in moderate/severe health states after week 8 would discontinue ADHD treatment and remain in 
that state for the rest of the study period. Direct costs included drug wholesale acquisition costs and health state costs, all in \$US, year 2010 values. Utility associated with each health state was obtained from the literature and disutilities associated with adverse events were applied for the first 4 weeks. Oneway sensitivity analyses and probabilistic sensitivity analysis (PSA) were conducted by varying costs, utilities, adverse-event duration, and transition probabilities.

Results: Compared with maintaining existing stimulant monotherapy, adding GXR to existing stimulant monotherapy was associated with an incremental drug cost of \$US1016 but a lower medical cost of \$US124, resulting in a total incremental cost of \$US892 at 1 year. The addition of GXR to stimulants led to an incremental QALY of 0.03 and an incremental cost-effectiveness ratio (ICER) of \$US31 660/QALY. In one-way sensitivity analysis, ICER values ranged from $\$$ US19723, when 100\% of patients were assumed to be severe in their initial health state, to \$US46 631, when the last observed states from the clinical trial were carried forward to the end of the 1-year analysis period. PSA demonstrated a 94.6\% likelihood that the ICER falls below \$US50 000/QALY.

Conclusions: The impairment associated with residual ADHD symptoms after stimulant therapy is becoming increasingly recognized. This is the first analysis of the cost effectiveness of stimulants combined with an adjunctive medication. This study suggests that the adjunctive therapy of GXR with stimulants is a cost-effective treatment based on a willingness-to-pay threshold of \$US50 000/QALY. This may address an unmet need among patients with suboptimal response to stimulant monotherapy.

\section{Key points for decision makers}

- Some paediatric attention-deficit hyperactivity disorder (ADHD) patients receiving stimulant treatment alone present with suboptimal symptom control

- Adjunctive treatment with guanfacine extended release (GXR) addresses an unmet need of this group of ADHD patients by improving their symptom control

- Adjunctive treatment with GXR in this ADHD patient population appears cost effective, addressing an unmet need among ADHD patients with suboptimal response to stimulant monotherapy

\section{Background}

Attention-deficit hyperactivity disorder (ADHD) is a common psychiatric disorder in childhood and adolescence, affecting $3-7 \%$ of school-age children in the US. ${ }^{[1,2]}$ ADHD is characterized by symptoms of hyperactivity, impulsivity and inattention, which interfere with a child's ability to function at school and at home. ${ }^{[3,4]}$
ADHD substantially impairs the quality of life of affected children and adolescents as well as their family members. ${ }^{[5]}$ It also poses considerable economic burden to the healthcare system and society worldwide. In the US, total healthcare costs for children and adolescents with ADHD have been estimated to be \$US7.9 billion dollars per year (year 2005 values). ${ }^{[6]}$ The indirect costs for ADHD related to education, criminality and 
caregiver work loss are even more striking. ${ }^{[6-8]}$ Costs associated with education and criminality among school-aged children with ADHD have been estimated to be \$US34.6 billion per year (year 2005 values). In addition, adult family members of children and adolescents with ADHD may incur an additional \$US4.9 billion in indirect costs annually (year 2000 values). ${ }^{[7]}$ In the Netherlands, mean 1-year costs per patient with ADHD were $€ 2040$ and the mean excess annual indirect cost due to absence from and decreased productivity at work of mothers of ADHD patients was $€ 1835$ (year 2004 values) ${ }^{[9]}$ In Belgium, annual private medical costs for a child with ADHD are more than six times the costs for a sibling control; annual societal medical costs for a child with ADHD are double the costs for a sibling control. ${ }^{[10]}$ In addition, in some countries, such as Germany, treatment costs associated with ADHD have been increasing. ${ }^{[1]}$

Effective treatment of ADHD may reduce the psychosocial and economic burden associated with the disease. Disease management usually involves some combination of behaviour modification, lifestyle changes, counselling and medication. Stimulants, such as amphetamine and methylphenidate, are the mainstays of pharmacological therapy and are first-line treatment options for the majority of patients with ADHD. ${ }^{[3]}$ However, based on a systematic literature review and pooling of randomized controlled trials of children aged 6-12 years, on average, approximately $30 \%$ of children with ADHD do not have an adequate response to the stimulant methylphenidate. ${ }^{[12]}$ Patients with suboptimal response to stimulants present a unique challenge in the management of ADHD. Alternative treatment options may include non-stimulants indicated for ADHD (e.g. atomoxetine, guanfacine extended release [GXR] or clonidine extended release) and some medications that are not approved by the US FDA for ADHD (such as tricyclic antidepressants, bupropion, guanfacine immediate release and clonidine immediate release), as well as adding adjunctive pharmacotherapy to the existing stimulants. Adjunctive therapy may help further improve the core ADHD symptoms that cannot be fully controlled by stimulants alone. Therefore, it could be a practical treatment option among patients with suboptimal response to stimulants. ${ }^{[13]}$ While information regarding the use of adjunctive therapy with stimulants in children/adolescents with ADHD is scarce, a retrospective claims-based analysis found that $21 \%$ of adult patients on longacting stimulants used adjunctive therapy for the treatment of ADHD. ${ }^{[14]}$ Despite the relatively high prevalence of adjunctive therapy among stimulant users, there are limited clinical trial data to support the combination use of a stimulant and another medication. In fact, many commonly used adjunctive medications, such as bupropion, clonidine immediate release and guanfacine immediate release, are not approved for the treatment of ADHD by the FDA.

To date, the only once-daily FDA-approved medication for adjunctive therapy to stimulants is GXR. GXR is not yet approved in the EU; however, there are two clinical trials currently ongoing in the EU. ${ }^{[15,16]}$ GXR is a selective alpha-2A adrenergic receptor agonist and was approved by the FDA in September 2009 as monotherapy for the treatment of ADHD in children and adolescents aged 6-17 years. The mechanism of action of guanfacine in the treatment of ADHD is unknown. Preclinical research suggests that guanfacine strengthens working memory and prefrontal cortex neuronal firing. In clinical trials, GXR has demonstrated efficacy and tolerability both as a monotherapy and as an adjunctive therapy to stimulants. ${ }^{[17-19]}$ In a phase II open-label trial of GXR as an adjunctive therapy to stimulants (methylphenidate or amphetamine), no unique adverse events (AEs) were observed with GXR given with a stimulant compared with those reported historically for either treatment alone. ${ }^{[18]}$ Recently, results from a phase III, double-blind, randomized, placebo-controlled, multicentre, doseoptimization study evaluated the efficacy and tolerability of GXR as adjunctive therapy to stimulants in children and adolescents aged 6-17 years with ADHD and suboptimal response to stimulant monotherapy. ${ }^{[20,21]}$ Significant reductions in ADHD symptoms were reported for subjects receiving GXR, when dosed either in the morning or in the evening, in addition to the existing stimulant, compared with maintaining the existing stimulant in the same patient population. ${ }^{[20,21]}$ 
The cost effectiveness of GXR as an adjunctive therapy to stimulants has not been explored. While adding GXR to stimulants has the potential to increase pharmacy costs and AEs associated with ADHD treatment due to the need for two pharmacological agents, it is associated with better clinical outcomes than stimulant monotherapy among patients with suboptimal response to stimulants. ${ }^{[20]}$ To fully understand the economic value of GXR as an adjunctive therapy, we conducted a cost-effectiveness analysis (CEA) of GXR as an adjunctive therapy to existing stimulants compared with maintaining existing stimulant monotherapy among children and adolescents with ADHD who had a suboptimal response to existing stimulants.

\section{Methods}

\section{Model Overview}

We designed a Markov model to evaluate the cost effectiveness of GXR as an adjunctive therapy to stimulants compared with stimulant monotherapy in the treatment of ADHD in children and adolescents with suboptimal response to stimulants. The model was created from a US third-party payer perspective with a 1-year time horizon, which has been commonly used in other CEAs of ADHD treatments. ${ }^{[12,22,23]}$

Patient-level data from the phase III randomized, double-blind clinical trial comparing GXR as adjunctive therapy to stimulants with placebo plus stimulants were used to estimate the transition probabilities among health states, the initial distribution of health states, and the AE rates for each treatment arm. ${ }^{[20,21,24]}$ In addition, data extracted from a literature review provided inputs for utilities, medical costs and disutilities associated with AEs.

Phase III Trial of Guanfacine Extended Release as an Adjunctive Therapy to Stimulants

The phase III, 9-week, double-blind, randomized, placebo-controlled, multicentre, dose-optimization study evaluated the efficacy and safety of GXR $(1,2,3$ and $4 \mathrm{mg} /$ day $)$ plus stimulants compared with placebo plus stimulants in children and adolescents with ADHD aged 6-17 years with suboptimal response to stimulants. ${ }^{[21]}$ Suboptimal response to stimulants was defined as treatment with a stable dose of stimulant for at least 4 weeks with improvement; however, mild to moderate ADHD symptoms must have remained present (an ADHD Rating Scale Version IV [ADHD-RS-IV] score of $\geq 24$ and a Clinical Global ImpressionSeverity [CGI-S] score of $\geq 3$ ) or an investigator assessment of inadequate response to current psychostimulant must have been given. ${ }^{[20,21]}$ Subjects with no response to stimulants prior to study enrolment were excluded from participating in this study. The study included a 5-week dose-optimization period for GXR, followed by a 3 -week dose-maintenance period and a 1-week dose-tapering period. This design took into account the prompt onset of effects for most ADHD medications. ${ }^{[21]}$

In the trial, a total of 461 patients were randomized; however, only 455 of the randomized patients received at least one dose of study medication and constituted the full analysis set (FAS)/safety population: 150 patients received GXR in the morning, 152 received GXR in the evening, and 153 received placebo, all of whom continued their stable dose of stimulant throughout the trial. ${ }^{[21]}$ The FAS/safety population dataset was used in this study.

The primary efficacy outcome was a change in ADHD-RS-IV total score from baseline to endpoint, defined as the last on-therapy, postbaseline treatment week prior to any dose taper at which a valid ADHD-RS-IV total score was collected. Secondary outcomes included the CGI-S and AE outcomes. ${ }^{[21]}$

The mean age of the trial population was 10.8 years. The trial population was $71.6 \%$ male. ${ }^{[21]}$ The majority of study subjects were in the moderate health state (see the Model Description section for a description of the health states) at baseline, accounting for $90.5 \%$ of the total trial population. Patients in the mild and severe states accounted for $3.5 \%$ and $5.9 \%$, respectively. ${ }^{[24]}$

To be consistent with the GXR package insert, this study compared the combined GXR treatment arms (evening and morning) with the placebo arm. ${ }^{[20]}$ 


\section{Model Description}

The Markov model consisted of four health states reflecting the severity of ADHD symptoms - normal, mild, moderate and severe (figure 1) with a cycle length of 1 week. The cycle length reflected the frequency of efficacy assessment in the phase III trial. The health states were defined based on the CGI-S. The CGI-S is a 7-point scale designed for clinicians to rate the severity of a patient's condition and is a common endpoint in clinical trials evaluating new treatments for ADHD. It ranges from 1 ('not ill at all') to 7 ('among the most extremely ill subjects'). In our study, the normal state was defined as a CGI-S score of 1, i.e. 'not ill at all'; the mild state was defined as a CGI-S score of 2 or 3 , i.e. 'borderline ill' or 'mildly ill'; the moderate state corresponded to a CGI-S score of 4 or 5, i.e. 'moderately ill' or 'markedly ill'; and lastly, the severe state was defined as a CGI-S score of 6 or 7, i.e. 'severely ill' or 'among the most extremely ill subjects'. Stratification with four health states could better differentiate patients by level of symptoms than with two health states (i.e. response vs non-response), which have been used in previous ADHD costeffectiveness literature. ${ }^{[22,25]}$ Because the four health states have not been defined in the literature based on the ADHD-RS-IV scale, the CGI-S was used in this study in order to be consistent with the definition of health states used in the study from which ADHD health utility values were extracted. ${ }^{[26]}$

A hypothetical cohort of children and adolescents with ADHD received GXR plus stimulants or placebo plus stimulants at the model entry and transitioned between the four health states at the beginning of each cycle. The initial distribution among the health states was determined based on the observed distribution of the phase III trial population at baseline. The model consisted of two stages. The first stage was modelled to span from week 0 to week 8 , and the second stage spanned from week 9 to week 52. All patients continued their assigned treatments during the first stage. In the second stage, patients in the moderate or severe states were considered to be non-responsive and thus permanently discontinued the treatments. Because most of the patients in the trial were in the moderate or severe state at baseline,

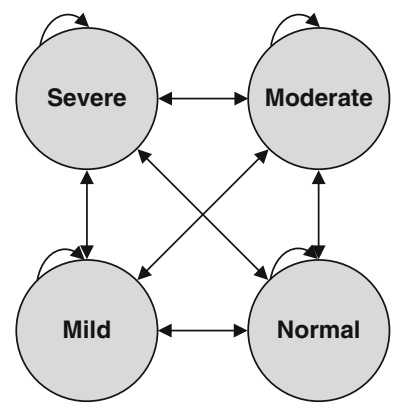

Combined GXR plus stimulants [mean (SE)]

\begin{tabular}{|l|c|c|c|c|}
\hline To & Normal & Mild & Moderate & Severe \\
\hline \multirow{2}{*}{ Normal } & $\begin{array}{c}0.766 \\
(0.041)\end{array}$ & $\begin{array}{c}0.231 \\
(0.041)\end{array}$ & $\begin{array}{c}0.003 \\
(0.001)\end{array}$ & $\begin{array}{c}0.000 \\
(0.000)\end{array}$ \\
\hline \multirow{2}{*}{ Mild } & 0.112 & 0.817 & 0.070 & 0.000 \\
& $(0.014)$ & $(0.016)$ & $(0.011)$ & $(0.000)$ \\
\hline \multirow{2}{*}{ Moderate } & 0.003 & 0.258 & 0.737 & 0.002 \\
& $(0.001)$ & $(0.019)$ & $(0.019)$ & $(0.001)$ \\
\hline \multirow{2}{*}{ Severe } & 0.000 & 0.000 & 0.361 & 0.639 \\
& $(0.000)$ & $(0.000)$ & $(0.102)$ & $(0.102)$ \\
\hline
\end{tabular}

Placebo plus stimulants [mean (SE)]

\begin{tabular}{|l|c|c|c|c|}
\hline From & Normal & Mild & Moderate & Severe \\
\hline \multirow{2}{*}{ Normal } & $\begin{array}{c}0.759 \\
(0.056)\end{array}$ & $\begin{array}{c}0.238 \\
(0.055)\end{array}$ & $\begin{array}{c}0.003 \\
(0.001)\end{array}$ & $\begin{array}{c}0.000 \\
(0.000)\end{array}$ \\
\hline \multirow{2}{*}{ Mild } & 0.081 & 0.826 & 0.093 & 0.000 \\
& $(0.013)$ & $(0.017)$ & $(0.014)$ & $(0.000)$ \\
\hline \multirow{2}{*}{ Moderate } & 0.002 & 0.192 & 0.801 & 0.004 \\
& $(0.001)$ & $(0.015)$ & $(0.015)$ & $(0.003)$ \\
\hline \multirow{2}{*}{ Severe } & 0.000 & 0.000 & 0.236 & 0.763 \\
& $(0.000)$ & $(0.000)$ & $(0.068)$ & $(0.068)$ \\
\hline
\end{tabular}

Fig. 1. Diagram of health states. Patients may enter the model in the mild, moderate or severe states. The data in the tables represent the various transition probabilities. $\mathbf{G X R}=$ guanfacine extended release; $\mathbf{S E}=$ standard error. 
being in the moderate or severe state at week 8 would indicate a lack of response to treatment. The two-stage assumption was consistent with the approach used in the technology assessment conducted by the National Institute for Health and Clinical Excellence (NICE), ${ }^{[22]}$ which assumed that children who did not respond to the initial therapy by week 8 would not continue the original treatment. In addition, the assumption was also supported by a real-world adherence study, which showed that the majority of patients with ADHD were compliant with treatment for an average of 2 months. ${ }^{[27]}$

\section{Model Assumptions}

The assumptions made were as follows:

- Modelled patients were in one of the four health states in each cycle (normal, mild, moderate or severe) and could transition into a less or more severe state at the beginning of each cycle of the model.

- Patients followed the transition probabilities during the trial period as long as they were on treatment.

- Patients who transitioned into the moderate or severe state during the second stage discontinued treatment and remained in the last health state while on treatment for the rest of the model period.

- Patients who discontinued treatment remained off treatment and did not switch to new treatments. Therefore, the model assumed that these patients only incurred medical costs for the rest of the model period. Subsequent therapies were not included in the model for two reasons: (i) there was insufficient clinical evidence or consensus on how patients would be treated after failing combination therapy; and (ii) choices of subsequent therapies would be different for the two treatment arms. For example, patients in the stimulant monotherapy arm could use GXR as an adjunctive therapy while this was not an option for patients in the GXR plus stimulants arm. If different subsequent therapies were assumed for the two comparison arms, the final outcomes would reflect the effects of both the initial therapy and subsequent thera- pies, which was not the objective of the current study.

- The health utility corresponding to each health state did not depend on treatment, i.e. regardless of whether a patient was on treatment or not and the type of treatment, patients in the same health state had the same health utility.

\section{Model Inputs}

\section{Transition Probabilities}

Transition probabilities were calculated based on patient-level data from the phase III trial. Following the trial definition of the endpoint, we used the efficacy data from the first 8 weeks. The weekly endpoint measure was defined as in the trial, as the last on-therapy, post-randomization treatment week, prior to any dose taper, at which a valid ADHD-RS-IV total score was collected. Patients were assigned each week to one of the four health states from week 0 to week 8 based on the observed weekly CGI-S values. ${ }^{[24]}$ To be consistent with the trial protocol, missing values were imputed using the last observation carried forward (LOCF).

In the base-case model, we used ordered logit models to estimate the transition probabilities, where the dependent variable was the current health state and the independent variable was the health state in the previous week. By estimating transition probabilities in this way, we assumed that health status in one state was purely a function of health state in the previous state. In addition, patients' transitions between health states in the eight time periods were assumed to be constant (e.g. time independent). Transition probabilities were estimated for the placebo plus stimulants arm and the combined GXR plus stimulants arm. The estimated transition probabilities were applied throughout the entire 52-week model period for patients remaining on treatment (see figure 1).

\section{Costs}

Because the CEA was conducted from a US third-party payer perspective, only direct costs were estimated, which included treatment drug costs and medical costs. All cost inputs were extracted from public data or literature and were 
Table I. Key model inputs

\begin{tabular}{lll}
\hline Model input & Value & Source \\
\hline Initial health state distribution (\%) & & \\
Normal & 0.00 & Phase III trial[ ${ }^{24]}$ \\
Mild & 3.52 & \\
Moderate & 90.55 & \\
Severe & 5.93 &
\end{tabular}

\section{Weekly medical costs (\$US) ${ }^{\mathrm{a}}$}

$\begin{array}{lll}\text { Normal } & 7.19 \quad \text { Guevara et al. }{ }^{[2]} \\ \text { Mild } & 18.15 \\ \text { Moderate } & 30.79 \\ \text { Severe } & 43.44 \\ & \\ \text { Utility inputs } & \\ \text { Normal } & 0.839 \quad \text { Lloyd et al. }{ }^{[26]} \\ \text { Mild } & 0.787 \\ \text { Moderate } & 0.578 \\ \text { Severe } & 0.444\end{array}$

WAC price of ADHD medication per pill (\$US) ${ }^{\mathrm{a}}$

$\begin{array}{lll}\text { Intuniv }^{\circledR b} & 4.95 & \text { Medi-Span }^{\circledast[30]} \\ \text { Adderall XR }^{\circledR C} & 6.53 & \\ \text { Concerta }^{\circledR \mathrm{d}} & 5.83 \\ \text { Focalin XR }^{\circledR e} & 4.92 \\ \text { Metadate CD }^{\circledR f} & 4.70 \\ \text { Ritalin LA }^{\oplus \mathrm{g}} & 4.23 \\ \text { Vyvanse }^{\circledR \mathrm{h}} & 4.88\end{array}$

AWP of ADHD medication per pill (\$US) ${ }^{\mathrm{a}}$

$\begin{array}{ll}\text { Intuniv }^{\circledR b} & 5.94 \\ \text { Adderall XR }^{\circledast \mathrm{C}} & 7.84 \\ \text { Concerta }^{\circledR \mathrm{d}} & 7.00 \\ \text { Focalin XR }^{\oplus \mathrm{e}} & 5.90 \\ \text { Metadate CD }^{\oplus \mathrm{f}} & 5.64 \\ \text { Ritalin LA }^{\oplus \mathrm{g}} & 5.08 \\ \text { Vyvanse }^{\circledast \mathrm{h}} & 5.85\end{array}$

\section{Percentage of patients taking stimulant (\%)}

$\begin{array}{lll}\text { Adderall } X R^{\circledR C} & 17.80 & \text { Phase III trial[ }{ }^{[21]} \\ \text { Concerta }^{\circledR \mathrm{d}} & 45.27 & \\ \text { Focalin } \mathrm{XR}^{\circledR \mathrm{e}} & 5.93\end{array}$

Continued
Table I. Contd

\begin{tabular}{|c|c|c|}
\hline \multicolumn{2}{|c|}{ Model input } & Value Source \\
\hline \multicolumn{2}{|c|}{ Metadate $C D^{\circledR f}$} & 1.10 \\
\hline \multicolumn{2}{|c|}{ Ritalin LA ${ }^{\oplus g}$} & 0.44 \\
\hline \multicolumn{2}{|c|}{ Vyvanse $^{\circledR h}$} & 29.45 \\
\hline \multicolumn{3}{|c|}{ a Costs are in year 2010 values. } \\
\hline \multicolumn{3}{|c|}{ b Intuniv ${ }^{\circledR}$ : GXR. } \\
\hline \multicolumn{3}{|c|}{$\begin{array}{l}\text { C Adderall } \mathrm{XR}^{\circledR} \text { : mixed salts of a single-entity amphetamine } \\
\text { product extended release. }\end{array}$} \\
\hline \multicolumn{3}{|c|}{ d Concerta $^{\circledR}$ : methylphenidate $\mathrm{HCl}$. } \\
\hline \multicolumn{3}{|c|}{ e Focalin $\mathrm{XR}^{\oplus}$ : dexmethylphenidate $\mathrm{HCl}$ extended release. } \\
\hline \multicolumn{3}{|c|}{ f $\quad$ Metadate $\mathrm{CD}^{\oplus}$ : methylphenidate $\mathrm{HCl}$, USP, controlled delivery. } \\
\hline \multicolumn{3}{|c|}{ g Ritalin $\mathrm{LA}^{\oplus}$ : methylphenidate $\mathrm{HCl}$ long acting. } \\
\hline \multicolumn{3}{|c|}{ h Vyvanse $^{\circledR}$ : lisdexamfetamine dimesylate. } \\
\hline
\end{tabular}

$\mathbf{A D H D}=$ attention-deficit hyperactivity disorder; $\mathbf{A W P}=$ average wholesale price; $\mathbf{G X R}=$ guanfacine extended release; $\mathbf{H C l}=$ hydrochloride; USP $=$ United States Pharmacopeia; $\mathbf{W A C}=$ wholesale acquisition cost.

inflation-adjusted to \$US, year 2010 values using the medical care component of the Consumer Price Index ${ }^{[28]}$ (table I).

The wholesale acquisition cost (WAC) ${ }^{[30]}$ was used as the unit cost for GXR and stimulants. If a drug had different doses, the average WAC among all available doses was estimated. The stimulants used in the model were consistent with what was observed in the phase III trial, which included Adderall $\mathrm{XR}^{\circledR}$ (mixed salts of a single-entity amphetamine product extended release; Shire Pharmaceuticals, Inc., Wayne, PA, USA), Vyvanse ${ }^{\circledR}$ (lisdexamfetamine dimesylate; Shire Pharmaceuticals, Inc., Wayne, PA, USA), Concerta $^{\circledR}$ (methylphenidate hydrochloride [HCl]; OrthoMcNeil-Janssen Pharmaceuticals, Inc., Raritan, NJ, USA), Focalin $\mathrm{XR}^{\circledR}$ (dexmethylphenidate $\mathrm{HCl}$ extended release; Novartis Pharmaceuticals Corporation, East Hanover, NJ, USA), Ritalin $\mathrm{LA}^{\circledR}$ (methylphenidate $\mathrm{HCl}$ long acting; Novartis Pharmaceuticals Corporation, East Hanover, NJ, USA), and Metadate $\mathrm{CD}^{\circledR}$ (methylphenidate $\mathrm{HCl}$, United States Pharmacopeia [USP], controlled delivery; UCB, Inc.; Brussels, Belgium). Most patients in the trial were taking either Concerta $^{\circledR}(45 \%)$ or Vyvanse ${ }^{\circledR}(29 \%)$. The final unit cost for stimulants was estimated as a weighted 
average of WACs based on the distribution of stimulants observed in the trial. We assumed that patients took one dose of stimulants and GXR per day as per the trial design.

Medical costs by disease severity among patients with ADHD have not been studied. Medical cost inputs used for this study were derived based on a retrospective cohort study comparing healthcare costs between children with ADHD and those without ADHD in a health maintenance organization in western Washington state in the US. ${ }^{[29]}$ In this study, children had a mean age of 11.7 years and $77.2 \%$ were male. Of the patients with ADHD, 28.7\% had co-morbid mental health conditions (11.1\% internalizing conditions, $12.3 \%$ externalizing conditions, $3.6 \%$ substance abuse/dependence).

To account for the skewed distribution of healthcare costs and ensure that higher costs were assigned to more severe states, we assumed that the annual medical costs for patients in the normal state were the same as median medical costs for non-ADHD patients (\$US374); the annual medical costs for patients in the mild state were the same as the median medical costs for ADHD patients (\$US944); the annual medical costs for patients in the moderate state were the same as the mean medical costs for ADHD patients (\$US1601); and the annual medical costs for patients in the severe state were the ones for the moderate state plus the medical cost difference between the mild and moderate states (\$US2259). All annual costs were divided by 52 weeks to estimate costs per cycle (table I).

\section{Utilities}

Utilities Associated with Health States

Utility values were obtained from a recent study by Lloyd et al. ${ }^{[26]}$ In our model, health states based on the CGI-S were defined in the same way as those from Lloyd et al., with the exception that the severe state in their study excluded the most severe CGI-S state 7, 'among the most extremely ill subjects'. The utility data were collected from a survey of 100 members of the general public in the UK. Both a time trade-off (TTO) method and a visual analogue scale (VAS) were used to estimate the utilities associated with the four health states. Utility values estimated from the TTO method were used in this study. The means and standard errors of the utilities for normal, mild, moderate and severe states were $0.839(0.020)$, 0.787 (0.022), $0.578(0.028)$ and $0.444(0.023)$, respectively.

\section{Disutilities Associated with Adverse Events}

In addition to utility associated with each health state, disutilities associated with AEs were also applied. AEs were included if they impacted at least $5 \%$ of patients in any treatment arm and were listed on the product insert. ${ }^{[20]} \mathrm{AE}$ rates were obtained from observed rates for each treatment arm during the phase III trial (table II). ${ }^{[20,24]}$ This assumed that no new AEs would occur after the trial period. In the base case, disutilities associated with AEs were applied for the first 4 weeks after treatment initiation. Disutility values associated with these AEs were identified from a literature review. ${ }^{[31-34]}$ The total disutility values associated with these AEs were 0.265 and 0.123 for the GXR plus stimulants and stimulant monotherapy treatment arms, respectively.

\section{Model Outputs}

Model outputs included cost and effectiveness outcomes for each treatment arm, incremental cost, incremental effectiveness, and incremental cost-effectiveness ratio (ICER) comparing GXR plus stimulants with stimulant monotherapy. Total direct costs were estimated by summing direct costs across all model cycles. Both total direct costs and costs for each component (ADHD drug costs and medical costs) were estimated. Incremental costs were estimated as the cost differences per patient between the two treatment arms during the model period. QALYs were estimated as the sum of the weighted time spent in each cycle for the 1-year model period, using the utility for each cycle as the weight. Incremental effectiveness was estimated as the QALY difference per patient between the two treatment arms. Finally, the ICER was estimated as the ratio between incremental cost and incremental effectiveness and was expressed as an incremental cost per QALY. 
Sensitivity Analysis

To test the model's robustness, key parameters and model assumptions were varied using one-way sensitivity analyses on transition probabilities, costs, utilities, AE duration, stimulant choice, and initial state distribution.

- Transition probabilities: Instead of a modelling approach, we used the observed transitions between the health states during the first stage (i.e. the first 8 weeks). We further assumed that health states were stabilized without further transitions in the second stage (i.e. week 9 to week 52) and carried forward the week- 8 health states until the end of the model period.

- Drug costs: The average wholesale prices (AWPs) were used to estimate the unit costs for ADHD medications instead of the WACs. Furthermore, costs for stimulants were varied by assuming $100 \%$ of patients were on Ritalin $\mathrm{LA}^{\circledR}$, Adderall XR ${ }^{\circledR}$, Vyvanse ${ }^{\circledR}$, or Metadate $\mathrm{CD}^{\circledR}$.

- Medical costs: We varied the cost of each health state by setting the cost equal to that of the next less severe health state or the cost equal to that of the next more severe health state. For the lower bound of the cost for a normal patient, we used the cost of a normal patient minus $25 \%$. For the upper bound of the cost for a severe patient, we used the cost of a severe patient plus $25 \%$. We also considered a scenario where medical costs were assumed to be zero.

- Utility values: The utility values for the health states were varied to the upper and lower bounds of the $95 \%$ confidence intervals (CIs) reported in the study by Lloyd et al. ${ }^{[26]}$ The utilities corresponding to the upper bound of the $95 \%$ CI for the normal, mild, moderate and severe states were $0.880,0.830,0.633$ and 0.504 , respectively. The utilities corresponding to the lower bound of the $95 \%$ CI for the normal, mild, moderate and severe states were $0.799,0.744,0.523$ and 0.385 , respectively. ${ }^{[26]}$

- AEs: Disutilities associated with AEs were applied for 0, 2 and 8 weeks, respectively.

- Initial patient distribution: The initial distribution of patients was assumed to be $100 \%$ mild, $100 \%$ moderate and $100 \%$ severe.

A multivariate probabilistic sensitivity analysis (PSA) was also conducted with a Monte Carlo simulation of 1000 iterations. Model inputs with uncertainties were included in the PSA, including transition probabilities, the medical cost of each health state, the utility of each health state, and the duration of AEs. For transition prob-

Table II. Adverse events ${ }^{a}$

\begin{tabular}{|c|c|c|c|c|}
\hline$\overline{\mathrm{AE}}$ & GXR plus stimulants (\%) & Stimulants only (\%) & Disutility & Source \\
\hline Headache & 21 & 13 & 0.353 & Revicki and Wood ${ }^{[31]}$ \\
\hline Somnolence ${ }^{d}$ & 18 & 7 & 0.380 & Heeg et al. ${ }^{[32]}$ \\
\hline Insomnia ${ }^{e}$ & 12 & 6 & 0.324 & Botteman et al. ${ }^{[33]}$ \\
\hline Fatigue & 10 & 3 & 0.380 & Heeg et al. ${ }^{[32]}$ \\
\hline Abdominal pain ${ }^{\dagger}$ & 10 & 3 & 0.040 & Kristiansen et al..$^{[34]}$ \\
\hline Dizziness & 8 & 4 & 0.461 & Revicki and Wood ${ }^{[31]}$ \\
\hline Decreased appetite & 7 & 4 & 0.048 & Revicki and Wood ${ }^{[31]}$ \\
\hline Nausea & 5 & 3 & 0.048 & Revicki and Wood ${ }^{[31]}$ \\
\hline \multicolumn{5}{|c|}{$\begin{array}{l}\text { AEs that impacted at least } 5 \% \text { of any treatment arm were included. In the base case, AEs were assumed to result in utility decrements } \\
\text { lasting } 4 \text { weeks. }\end{array}$} \\
\hline \multicolumn{5}{|c|}{ b Disutility data were taken from the Tufts CEA Registry. ${ }^{[35]}$} \\
\hline \multicolumn{5}{|c|}{$\begin{array}{l}\text { c The disutility associated with fatigue was assumed to equal somnolence. The disutility from dizziness was assumed to equal } \\
\text { lightheaded/faint. The disutility from decreased appetite was assumed to equal nausea. }\end{array}$} \\
\hline \multicolumn{5}{|c|}{ d The somnolence term included somnolence, sedation and hypersomnia. } \\
\hline \multicolumn{5}{|c|}{ e The insomnia term included insomnia, initial insomnia and middle insomnia. } \\
\hline \multicolumn{5}{|c|}{ f The abdominal pain term included abdominal pain, abdominal pain upper and abdominal pain lower. } \\
\hline \multicolumn{5}{|c|}{$\mathbf{A E}=$ adverse event; $\mathbf{C E A}=$ cost-effectiveness analysis; $\mathbf{G X R}=$ guanfacine extended release.} \\
\hline
\end{tabular}


abilities, probabilities of remaining in the same health state were included as a random variable with Beta distribution bounded by 0 and 1 . Transition probabilities to other health states were re-estimated based on the ratios between these probabilities in the base case and the new probability of remaining in the same health state in the PSA. The calculations ensure that transition probabilities from a given health state to all four states sum to one. For medical costs and utilities, the inputs for the normal state were included as random variables. Gamma distribution was used for the cost variable assuming that the mean cost equalled the standard deviation; Beta distribution bounded by 0 and 1 was used for the utility variable. To ensure that medical costs increase with disease severity and utilities decrease with disease severity, the values for each other state (i.e. mild, moderate and severe) were re-estimated by applying the ratio between that state and the normal state in the base case to the new value for the normal state in the PSA. In addition, the durations of all AEs were varied simultaneously by randomly drawing from a Uniform distribution from 0 to 8 weeks. The ICER values at 1 year were plotted in a cost-effectiveness acceptability curve (CEAC), from which the probability of GXR plus stimulants being cost effective compared with placebo plus stimulants was assessed based on different willingness-to-pay (WTP) thresholds.

\section{Results}

\section{Base-Case Analysis}

Adding GXR as an adjunctive therapy to stimulants was associated with \$US1016 higher costs for ADHD pharmacy medication costs (\$US1690 vs \$US675) but \$US124 less in medical costs
(\$US1297 vs \$US1421), leading to a total incremental cost of \$US892 (\$US2987 vs \$US2095) compared with stimulant monotherapy. The combination therapy also increased QALYs by 0.028 (0.655 vs 0.627). Excluding the impact of AEs led to a QALY difference of 0.031 (0.660 vs 0.629 ). As a result, the ICER was \$US31 660/QALY for treatment with GXR as an adjunctive therapy to stimulants compared with stimulant monotherapy among children and adolescents with ADHD who had a suboptimal response to stimulants (table III).

\section{Sensitivity Analysis}

Results from the one-way sensitivity analyses showed that the ICER ranged from SUS19723/ QALY, when all patients were assumed to start in the severe state, to \$US46631/QALY, when transition probabilities as observed in the trial were applied and patients were assumed not to transition beyond week 8 (figure 2). Overall, the results were sensitive to the assumptions for transition probabilities, initial health state distribution, and unit drug costs. All ICERs from the sensitivity analyses were within $+/-20 \%$ of the base-case value, with the exception of the following three assumptions: (i) transition probabilities as observed in the trial, (ii) $100 \%$ of patients starting with the severe health state, and (iii) AWPs as unit drug costs. When no medical cost offsets were assumed by using more efficacious treatment, the ICER increased from \$US31660/ QALY to \$US36065/QALY. The PSA demonstrated a $94.6 \%$ likelihood that the ICER was less than \$US50 000/QALY. The median ICER was shown to be \$US32 720/QALY with a 95\% CI of \$US17 338/QALY to \$US54 938/QALY (figure 3).

Table III. Base-case results

\begin{tabular}{lllllllll}
\hline & $\begin{array}{l}\text { Total costs } \\
(\$ \cup S)^{\mathrm{a}}\end{array}$ & $\begin{array}{l}\text { Drug costs } \\
(\$ \mathrm{U})^{\mathrm{a}}\end{array}$ & $\begin{array}{l}\text { Medical costs } \\
(\$ \mathrm{US})^{\mathrm{a}}\end{array}$ & $\begin{array}{l}\text { Average } \\
\text { QALYs }\end{array}$ & $\begin{array}{l}\text { QALYs } \\
\text { without AEs }\end{array}$ & $\begin{array}{l}\text { Incremental } \\
\text { total costs }\end{array}$ & $\begin{array}{l}\text { Incremental } \\
\text { QALYs }\end{array}$ & $\begin{array}{l}\text { ICER } \\
(\$ \mathrm{SU})\end{array}$ \\
\hline $\begin{array}{l}\text { Stimulants } \\
\text { only }\end{array}$ & 2095 & 675 & 1421 & 0.627 & 0.629 & Reference & Reference & Reference \\
$\begin{array}{l}\text { GXR plus } \\
\text { stimulants }\end{array}$ & 2987 & 1690 & 1297 & 0.655 & 0.660 & 892 & 0.028 & 31660 \\
\hline
\end{tabular}

a Costs are in year 2010 values.

$\mathbf{A E}=$ adverse event; $\mathbf{G X R}=$ guanfacine extended release; $\mathbf{I C E R}=$ incremental cost-effectiveness ratio. 


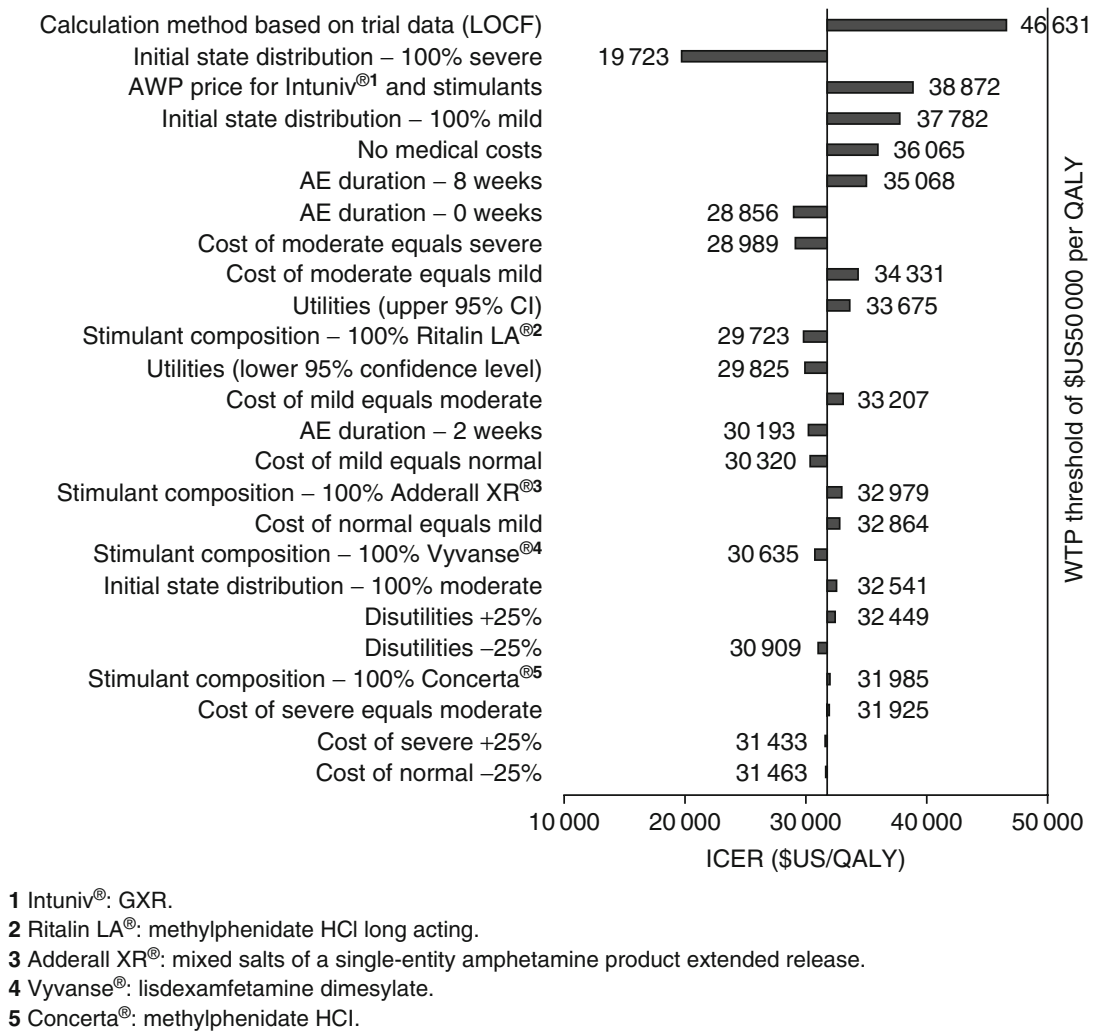

Fig. 2. Univariate sensitivity analysis. The base-case incremental cost-effectiveness ratio is $\$$ US31 $660 / Q A L Y$. $\mathbf{A E}=$ adverse event; $\mathbf{A W P}=$ average wholesale price; $\mathbf{C I}=$ confidence interval; $\mathbf{G X R}=$ guanfacine extended release; $\mathbf{H C I}=$ hydrochloride; $\mathbf{I C E R}=$ incremental cost-effectiveness ratio; $\mathbf{L O C F}=$ last observation carried forward; $\mathbf{W T P}=$ willingness to pay.

\section{Discussion}

The current CEA evaluated the cost effectiveness of GXR as an adjunctive therapy to stimulants compared with stimulant monotherapy among children and adolescents with ADHD who had suboptimal response to stimulants. The base-case analysis showed that although adding GXR as an adjunctive therapy increased ADHD drug costs, the costs could be partially offset by the difference in medical costs. In addition, the combination of GXR and stimulants led to, on average, 0.028 higher QALYs than stimulants alone. While the disutility associated with AEs was higher for those patients taking GXR and stimulants because of the added pharmacotherapy, this was offset by the increases in utility associated with symptom improvements. With the ICER of \$US31 660/QALY, GXR as an adjunctive therapy to stimulants would be considered as cost effective at a WTP threshold of \$US50000/QALY. The one-way sensitivity analyses conducted in our study found that the results were most sensitive to the assumptions about the transition probabilities. When the transition probabilities observed in the trial were used and patients were assumed not to transition beyond week 8, the model generated the highest ICER of \$US46 631/ QALY. This is not surprising as we assumed that the impact of the combination therapy was limited to only within the trial period - a conservative assumption against combination therapy. Other drivers of the results included the distribution of health states at the model entry and unit ADHD 
drug costs. However, none of the ICERs exceeded \$US50 000/QALY. It is worth mentioning that although assumptions had to be made to estimate medical costs associated with each health state, the results were not sensitive to these assumptions. Even if we completely eliminated the medical costs in the model estimation, the ICER was similar to the base-case value. While there is substantial debate in the US surrounding the use of cost effectiveness for decision making, CEAs are increasingly being used to support formulary reimbursement and tier status placement. ${ }^{[36]} \mathrm{A}$ review of published CEAs demonstrated that $34 \%$ mentioned an explicit threshold, with the median threshold of \$US50 000/QALY. ${ }^{[37]}$ However, recent research demonstrates that a threshold of \$US50 000/QALY is likely significantly lower than the WTP threshold within the US for a QALY, which is more likely between \$US100000 and \$US300 000. ${ }^{[38]}$ Therefore, the use of a \$US50 000/ QALY threshold can be seen as a lower bound for the value of a QALY, implying that even under conservative assumptions, GXR as an adjunctive therapy to stimulants is good value for the money. ${ }^{[39]}$

Suboptimal response to stimulants is not uncommon among children and adolescents with ADHD. ${ }^{[1,12,40]}$ These patients may be difficult to treat because they have fewer FDA-approved treat-

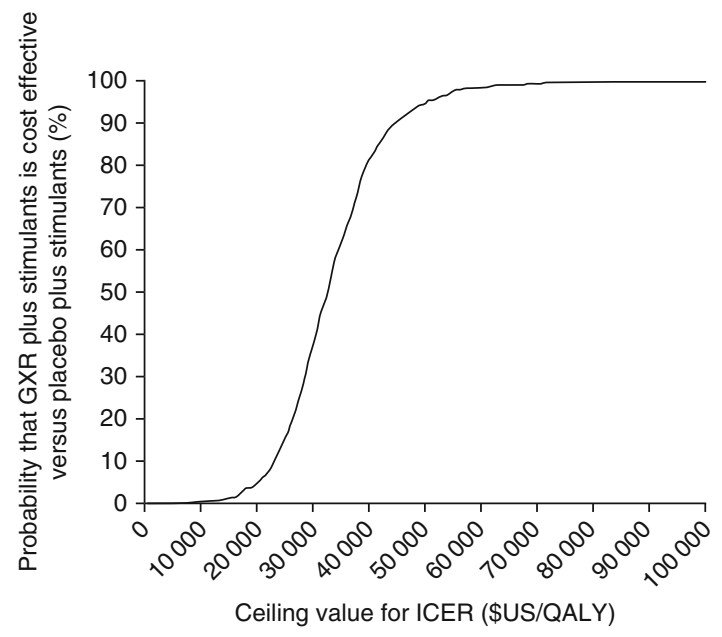

Fig 3. Cost-effectiveness acceptability curve comparing stimulant monotherapy with stimulant and adjunctive therapy of guanfacine extended release. $\mathbf{G X R}=$ guanfacine extended release; ICER= incremental cost-effectiveness ratio. ment options after stimulant therapy. The clinical evidence on the efficacy and safety of pharmacological treatment in suboptimal responders to stimulants is limited. GXR has demonstrated efficacy and tolerability among children and adolescents with ADHD and suboptimal response to stimulants. ${ }^{[17-19]}$ It is the only once-daily ADHD medication approved by the FDA as an adjunctive therapy to stimulants. The economic evidence on combination therapy is scarce. Given that GXR has recently received regulatory approval as an adjunctive therapy, the study provides timely information to physicians and payer decision makers about the economic value of adding GXR to stimulants.

\section{Comparison with Other Studies}

While many studies have evaluated the cost effectiveness of stimulants both compared with no treatment and compared with other stimulants, ${ }^{[12,22,25]}$ no study has evaluated the cost effectiveness of non-stimulants as an adjunctive therapy. Two studies have evaluated treatment strategies in patients with ADHD who had a suboptimal response to stimulants. A study by Faber et al. ${ }^{[41]}$ found that the stimulant osmoticrelease oral system (OROS) methylphenidate was a cost-effective treatment for patients in the Netherlands whose response to methylphenidate immediate release (MPH-IR) was suboptimal. This study differed from ours in a number of ways. First, the target population in the study by Faber et al. ${ }^{[41]}$ included patients with suboptimal response to MPH-IR, while the target population in our study consisted of patients with suboptimal response to a group of different stimulants, including amphetamine and methylphenidate, all of which are long-acting medications. Second, Faber et al. ${ }^{[41]}$ defined a suboptimal response as the suboptimal response due to patient non-compliance. Third, the two studies were conducted in two countries with vastly different healthcare systems. Therefore, the results between the two studies are not directly comparable. A separate study by Prasad et al. ${ }^{[23]}$ examined the cost effectiveness of atomoxetine compared with dexamfetamine in stimulant-failed patients in the UK. While this study 
analysed the cost effectiveness of a non-stimulant in a difficult-to-treat patient population, it did not consider atomoxetine as an adjunctive therapy to stimulants. In addition, the CEA in the study by Prasad et al. ${ }^{[23]}$ was conducted in a different target population, including patients who failed both MPH-IR and methylphenidate extended release, and in a different healthcare setting. These differences prevent us from directly comparing the results with those from our study.

\section{Limitations}

First, this economic evaluation used a Markov model framework. Markov models assume that a patient's disease course can be captured in transitions between different health states. While this may be an oversimplification of the real-world disease course, it is a commonly used approach and often necessary when the time horizon in the model extends beyond the clinical trial period. Although the Markov model used in this study may not fully reflect health states and transitions in the real world, it better differentiated ADHD severity than models used in previous CEAs, which only classified patients as responders or nonresponders. ${ }^{[22,25]}$ In addition, using the current model structure, the distribution of health states at week 8 was similar to the one observed in the phase III trial. ${ }^{[21]}$ In the placebo plus stimulants arm, the proportion of patients in the normal, mild, moderate and severe states based on the model estimation were $13.2 \%, 51.1 \%, 34.2 \%$ and $1.5 \%$, respectively, compared with $15.1 \%, 46.7 \%$, $36.2 \%$ and $2.0 \%$, respectively, as observed in the trial. In the GXR plus stimulants arm, the proportion of patients in the normal, mild, moderate and severe states based on the model estimation were $21.4 \%, 56.4 \%, 21.8 \%$ and $0.4 \%$, respectively, compared with $23.9 \%, 53.2 \%, 21.9 \%$ and $1.0 \%$, respectively, as observed in the trial. ${ }^{[21]}$

Second, other limitations involve the assumptions made in the model implementation. For example, we assumed that patients discontinued treatment if they transitioned into the moderate or severe state during the second stage. Although not all patients follow this treatment rule in the clinical setting, this may be a realistic assumption because most patients achieve optimal dosing within 8 weeks. If patients do not respond or lose response during an 8-week treatment, they may have limited benefit from continuing treatment and be considered for alternative therapies. Such an assumption was also implemented in a previous CEA of treatments for ADHD. ${ }^{[22]}$ In addition, we assumed that patients who discontinued treatment did not switch to other therapies. Although this assumption deviates from the clinical setting, it is necessary for our results to reflect only the outcome difference between the two treatments of interest and not be confounded by the subsequent therapies.

Third, the model estimation is limited by data availability. Because costs associated with different health states have not been reported, we had to make assumptions based on existing studies to estimate these model inputs. The decision rule for assigning cost values to each health state was relatively arbitrary. However, results from the one-way sensitivity analyses showed that the results were not sensitive to the assumptions used to estimate costs for each health state. Even if we conservatively assumed that all medical costs were zero, the ICER was still similar to the basecase value. Furthermore, data from different studies were applied in the model with the underlying assumption that the populations in these studies were comparable. However, the populations on which the model inputs were based were often different. To address this limitation, various sensitivity analyses were conducted to test the robustness of the results. Overall, our model results were not sensitive to cost and utility inputs.

Fourth, the study used data from the phase III clinical trial to estimate the effectiveness outcomes. To be enrolled in the clinical trial, patients were required to meet specific inclusion and exclusion criteria. For example, the trial excluded patients who have any current, controlled or uncontrolled, co-morbid psychiatric diagnosis (except oppositional defiant disorder). Of the patients in the FAS/safety population, $19.8 \%$ had a diagnosis of oppositional defiant disorder at baseline. ${ }^{[24]}$ The real-world effectiveness may be affected by patient compliance and co-morbidities. Therefore, the study findings may not be generalizable 
to a real-world setting. Future studies with estimated transition probabilities based on the realworld effectiveness data are needed. In addition, our study only considered patients taking longacting stimulants and therefore the results may not be generalizable to patients taking immediate-release stimulant formulations.

Finally, this study was conducted from a US third-party payer perspective and did not include indirect cost estimates associated with parent and caregiver work loss, additional educational resources, and crime and delinquency. A study by Pelham et al. ${ }^{[6]}$ found that healthcare costs associated with ADHD in children and adolescents account for approximately $19 \%$ of the total cost burden of ADHD; the remainder is associated with educational costs $(32 \%)$ and crime and delinquency costs $(50 \%)$. If the indirect costs were added into the model, then the ICER may have been even lower than the current result.

\section{Conclusions}

The impairment associated with residual ADHD symptoms after stimulant therapy and the need to optimize treatment are becoming increasingly recognized. This is the first analysis to estimate the cost effectiveness of stimulants combined with an adjunctive medication. The current economic evaluation indicates that GXR as an adjunctive therapy to stimulants is cost effective compared with stimulant monotherapy among children and adolescents with ADHD and a suboptimal response to stimulants. GXR as an adjunctive therapy could be a practical option to address the unmet needs of this patient population. In addition, findings from this study may better inform physicians and payer decision makers about the value of this new treatment for children and adolescents with ADHD, while assessing potential cost implications as well.

\section{Acknowledgements}

This study was funded by Shire Development LLC. Shire Pharmaceuticals, Inc is the manufacturer of Intuniv ${ }^{\circledR}$ (GXR). The authors independently conducted all analyses and wrote the manuscript. Shire Development LLC. provided the clinical trial data used in the analyses and preparation of the manuscript.
The authors controlled the decision to write and submit the manuscript for publication. VS, MHE and PH are employees of Shire Development LLC., and own stock in the company. JX, DM, MD and EQW are employees of Analysis Group, Inc.

The study was designed by VS, MHE, JX, DM, MD, PH and EQW. The methods were implemented by JX, DM and MD. VS, MHE, JX, DM, MD, PH and EQW participated in the review and interpretation of the data analysis. The manuscript was written by VS, JX, DM, MD and PH, and reviewed by MHE and EQW. VS is the guarantor for the overall content of this paper.

This paper is part of a theme issue co-edited by Lisa Prosser, University of Michigan, USA, and no external funding was used to support the publication of this theme issue.

\section{References}

1. Olfson M, Gameroff MJ, Marcus SC, et al. National trends in the treatment of attention deficit/hyperactivity disorder. Am J Psychiatry 2003 Jun; 160 (6): 1071-7

2. Wolraich ML, Hannah JN, Pinnock TY, et al. Comparison of diagnostic criteria for attention-deficit hyperactivity disorder in a county-wide sample. J Am Acad Child Adolesc Psychiatry 1996 Mar; 35 (3): 319-24

3. National Collaborating Centre for Mental Health. Attention deficit hyperactivity disorder: diagnosis and management of ADHD in children, young people and adults [NICE clinical guideline no. 72]. Leicester: British Psychological Society, 2009 Mar

4. American Psychiatric Association. Diagnostic and statistical manual of mental disorders. 4th ed. [text revision]. Washington, DC: American Psychiatric Association, 2000

5. Wehmeier PM, Schacht A, Barkley RA. Social and emotional impairment in children and adolescents with ADHD and the impact on quality of life. J Adolesc Health 2010 Mar; 46 (3): 209-17

6. Pelham WE, Foster EM, Robb JA. The economic impact of attention-deficit/hyperactivity disorder in children and adolescents. J Pediatr Psychol 2007 Jul; 32 (6): 711-27

7. Birnbaum HG, Kessler RC, Lowe S, et al. Costs of attention deficit-hyperactivity disorder (ADHD) in the US: excess costs of persons with ADHD and their family members in 2000. Curr Med Res Opin 2005; 20 (2): 195-205

8. Matza LS, Paramore C, Prasad M. A review of the economic burden of ADHD. Cost Eff Resour Alloc 2005; 3: 5

9. Hakkaart-van Roijen L, Zwirs BWC, Bouwmans C, et al. Societal costs and quality of life of children suffering from attention deficient hyperactivity disorder (ADHD). Eur Child Adolesc Psychiatry 2007; 16: 316-26

10. De Ridder A, De Graeve D. Healthcare use, social burden and costs of children with and without ADHD in Flanders, Belgium. Clin Drug Invest 2006; 26 (2): 75-90

11. Wehmeier PM, Schacht A, Rothenberger A. Change in the direct cost of treatment for children and adolescents with hyperkinetic disorder in Germany over a period of four years. Child Adolesc Psychiatry Ment Health 2009; 3 (1): 3

12. Gilmore A, Milne R. Methylphenidate in children with hyperactivity: review and cost-utility analysis. Pharmacoepidemiol Drug Saf 2001 Mar-Apr; 10 (2): 85-94

13. Adler LA, Reingold LS, Morrill MS, et al. Combination pharmacotherapy for adult ADHD. Curr Psychiatry Rep 2006; 8: 409-15 
14. Pohl GM, Van Brunt DL, Ye W, et al. A retrospective claims analysis of combination therapy in the treatment of adult attention-deficit/hyperactivity disorder (ADHD). BMC Health Serv Res 2009; 9: 95

15. Shire Development LLC. Efficacy and safety of extendedrelease guanfacine hydrochloride in children and adolescents aged 6-17 years with attention-deficit/hyperactivity disorder (ADHD) [ClinicalTrials.gov identifier: NCT01244490]. Bethesda (MD): National Library of Medicine, 2012 [online]. Available from URL: http://clinicaltrials.gov/ct2/show/ NCT01244490 [Accessed 2012 Jun 18]

16. Shire Development LLC. Maintenance of efficacy of extended-release guanfacine $\mathrm{HCl}$ in children and adolescents with attention-deficit/hyperactivity disorder (ADHD) [ClinicalTrials.gov identifier: NCT01081145]. Bethesda (MD): National Library of Medicine, 2012 [online]. Available from URL: http://clinicaltrials.gov/ct2/show NCT01081145 [Accessed 2012 Jun 18]

17. Biederman J, Melmed RD, Patel A, et al. A randomized, double-blind, placebo-controlled study of guanfacine extended release in children and adolescents with attentiondeficit/hyperactivity disorder. Pediatrics 2008 Jan; 121 (1): e73-84

18. Spencer TJ, Greenbaum M, Ginsberg LD, et al. Safety and effectiveness of coadministration of guanfacine extended release and psychostimulants in children and adolescents with attention-deficit/hyperactivity disorder. J Child Adolesc Psychopharmacol 2009 Oct; 19 (5): 501-10

19. Sallee F, McGough J, Wigal T, et al., on behalf of the SPD503 Study Group. Guanfacine extended release in children and adolescents with attention-deficit/hyperactivity disorder: a placebo-controlled trial. J Am Acad Child Adolesc Psychiatry 2009 Feb; 48 (2): 155-65

20. Intuniv ${ }^{\circledR}$ (guanfacine) extended-release tablets [package insert]. Wayne (PA): Shire US Inc., 2011 Feb

21. Wilens T, Bukstein O, Brams M, et al. A controlled trial of extended-release guanfacine and psychostimulants for attention-deficit/hyperactivity disorder. J Am Acad Child Adolesc Psychiatry 2012; 51 (1): 74-85

22. King S, Griffin S, Hodges Z, et al. A systematic review and economic model of the effectiveness and cost-effectiveness of methylphenidate, dexamfetamine and atomoxetine for the treatment of attention deficit hyperactivity disorder in children and adolescents. Health Technol Assess 2006; 10 (23): iii-iv, xiii-146

23. Prasad S, Arellano J, Steer C, et al. Assessing the value of atomoxetine in treating children and adolescents with ADHD in the UK. Int J Clin Pract 2009 Jul; 63 (7): 1031-40

24. Shire US Inc. A phase III, double-blind, randomized, placebocontrolled, multi-center, dose-optimization study evaluating the efficacy and safety of SPD503 in combination with psychostimulants in children and adolescents aged 6-17 years with a diagnosis of attention-deficit/hyperactivity disorder (ADHD). Shire US Inc., 2005. (Data on file)

25. Lord J, Paisley S. The clinical effectiveness and cost effectiveness of methylphenidate for hyperactivity in childhood [version 2]. London: National Institute for Clinical Excellence, 2000 Aug

26. Lloyd A, Hodgkins P, Sasane R, et al. Estimation of utilities in attention-deficit hyperactivity disorder for economic evaluations. Patient 2011; 4 (4): 247-57
27. Perwien A, Hall J, Swensen A, et al. Stimulant treatment patterns and compliance in children and adults with newly treated attention-deficit/hyperactivity disorder. J Manag Care Pharm 2004; 10 (2): 122-9

28. U.S. Bureau of Labor Statistics. Consumer price index: CPI tables [online]. Available from URL: http://www.bls.gov/ cpi [Accessed 2012 Jun 18]

29. Guevara J, Lozano P, Wickizer T, et al. Utilization and cost of health care services for children with attention-deficit/ hyperactivity disorder. Pediatrics $2001 \mathrm{Jul}$; 108 (1): 71-8

30. Medi-Span ${ }^{\circledR}$. Indianapolis (IN): Wolters Kluwer, 2011

31. Revicki D, Wood M. Patient-assigned health state utilities for depression-related outcomes: differences by depression severity and antidepressant medications. J Affect Disord 1998 Feb; 48 (1): 25-36

32. Heeg B, Buskens E, Botteman M, et al. The costeffectiveness of atypicals in the UK. Value Health 2008 Dec; 11 (7): 1007-21

33. Botteman MF, Foley DJ, Ozminkowski RJ, et al. Cost effectiveness of long-term treatment with eszopiclone for primary insomnia in adults: a decision analytical model. CNS Drugs 2007; 21 (4): 319-34

34. Kristiansen IS, Kvien TK, Nord E. Cost effectiveness of replacing diclofenac with a fixed combination of misoprostol and diclofenac in patients with rheumatoid arthritis. Arthritis Rheum 1999 Nov; 42 (11): 2293-302

35. Center for the Evaluation of Value and Risk in Health. The cost-effectiveness analysis registry. Boston (MA): Tufts Medical Center [online]. Available from URL: http:// www.cearegistry.org [Accessed 2012 Jun 18]

36. Bell C. The pursuit of transparency and quality improvement in cost-effectiveness analysis: a case study in diseasemodifying drugs for the treatment of multiple sclerosis. J Manag Care Pharm 2011; 17 (6): 463-8

37. Neumann PJ, Sandberg EA, Bell CM, et al. Are pharmaceuticals cost-effective? A review of the evidence. Health Affairs 2000; 19 (2): 92-109

38. Braithwaite RS, Meltzer DO, King JT, et al. What does the value of modern medicine say about the $\$ 50,000$ per quality-adjusted life-year decision rule? Med Care 2008; 46 : 349-56

39. Weinstein M. How much are Americans willing to pay for a quality-adjusted life year? Med Care 2008; 46 (4): 343-5

40. Swanson JM, Kraemer HC, Hinshaw SP, et al. Clinical relevance of the primary findings of the MTA: success rates based on severity of ADHD and ODD symptoms at the end of treatment. J Am Acad Child Adolesc Psychiatry 2001 Feb; 40 (2): 168-79

41. Faber A, van Agthoven M, Kalverdijk LJ, et al. Long-acting methylphenidate-OROS in youths with attention-deficit hyperactivity disorder suboptimally controlled with immediate-release methylphenidate: a study of cost effectiveness in the Netherlands. CNS Drugs 2008; 22 (2): 157-70

Correspondence: Dr Vanja Sikirica, PharmD, MPH, Director, Global Health Economics and Outcomes Research, Shire Development LLC., 725 Chesterbrook Boulevard, Wayne, PA 19087, USA.

E-mail: vsikirica@shire.com 